\title{
GAS FLOWS, STAR FORMATION AND GALAXY EVOLUTION
}

\author{
John E. Beckman ${ }^{1,2}$, Emilio Casuso $^{1}$, Almudena Zurita ${ }^{3}$ and Mónica Relaño ${ }^{1}$ \\ ${ }^{1}$ Instituto de Astrofísica de Canarias, Spain, \\ ${ }^{2}$ Consejo Superior de Investigaciones Científicas, Spain \\ ${ }^{3}$ Universidad de Granada, Dept. de Física Teórica y del Cosmos, Granada, Spain
}

\begin{abstract}
In the first part of this article we show how observations of the chemical evolution of the Galaxy: G- and K-dwarf numbers as functions of metallicity, and abundances of the light elements, D, Li, Be and B, in both stars and the interstellar medium (ISM), lead to the conclusion that metal poor H I gas has been accreting to the Galactic disc during the whole of its lifetime, and is accreting today at a measurable rate, $\sim 2 \mathrm{M}_{\odot}$ per year across the full disc. Estimates of the local star formation rate (SFR) using methods based on stellar activity, support this picture. The best fits to all these data are for models where the accretion rate is constant, or slowly rising with epoch. We explain here how this conclusion, for a galaxy in a small bound group, is not in conflict with graphs such as the Madau plot, which show that the universal SFR has declined steadily from $z=1$ to the present day. We also show that a model in which disc galaxies in general evolve by accreting major clouds of low metallicity gas from their surroundings can explain many observations, notably that the SFR for whole galaxies tends to show obvious variability, and fractionally more for early than for late types, and yields lower dark to baryonic matter ratios for large disc galaxies than for dwarfs. In the second part of the article we use NGC 1530 as a template object, showing from Fabry-Pérot observations of its $\mathrm{H} \alpha$ emission how strong shear in this strongly barred galaxy acts to inhibit star formation, while compression acts to stimulate it.
\end{abstract}

Keywords: Galaxy: evolution, Galaxy: accretion, galaxies: ISM, galaxies: kinematics galaxies: star formation

\section{Introduction}

The role of mergers in galaxy evolution has become increasingly recognized recently, stimulated by the central role of CDM cosmology (Navarro, Frenk \& White 1994, 1995; Power et al. 2003). The importance of mergers was realized from purely observational arguments by Toomre \& Toomre (1972); they argued that as tidal encounters generate short lived features, to yield today's "peculiar" galaxies a population of binary galaxies with highly eccentric orbits 
is required. If these have a flat binding energy distribution, their merger rate must have declined with time as $t^{-5 / 3}$ so that the ten obviously merging objects in the New General Catalogue must be the tail end of 750 remnants (see also Toomre, 1977). Zepf \& Koo (1989), prior to the Hubble Deep Fields, and Abraham (1999) using their contents, inferred that galaxy pair density grows as $(1+z)^{3}( \pm 1)$, while Brinchmann et al. (1998) showed that irregular galaxies form $10 \%$ of the total at $z \sim 0.4$ and $30 \%$ at $z \sim 0.8$. Peculiar morphologies, characteristic of mergers and interactions dominate at high redshift. The route to ellipticals via major mergers of spirals was first explored by Toomre \& Toomre (1972), by Toomre (1977), and by Verraraghavan \& White (1985).

Although most studies focused on the more spectacular major mergers, studies of the Galaxy have brought out the importance of minor mergers and accretions. For example Gilmore \& Feltzing (2000) in a major review, using arguments based on metallicity and kinematics, discuss its structural components: bulge, thin disc, thick disc (Freeman 1993; Gilmore \& Wyse 1998), and stellar halo, showing that the thin disc and bulge are relatively young, while the thick disc and halo are relatively old. In particular the thick disc formed at a specific epoch, some 10 Gyr ago (Gilmore \& Wyse 1998) as a result of a minor merger, and the Galaxy is accreting material now as it disrupts its smaller neighbours (Lynden-Bell 1976; Mirabel 1982; Savage et al. 2000). Here we will point up a process which is playing a key role in the evolution of the Galaxy, but which tends to be overlooked: the accretion of gas clouds of sub-galactic mass. This must be important in galaxy groups, small clusters, and the outskirts of rich clusters in general. We will show here, as the main thrust of the article, that the Galactic accretion rate has not declined during the disc lifetime and while this may seem surprising, it is a natural consequence of a plausible model for the Local Group. To maintain the star formation rates (SFR's) observed in late type galaxies now a steady inflow of gas from outside the galaxy is maintained. A key difference between a gas-rich and a gas-poor galaxy is that the capture cross-section for gas cloud accretion is higher in the former; this alone could account for the lower mean SFR's seen in late type galaxies but also for their greater relative scatter. The distribution of star formation across a galaxy disc is also affected strongly by gas flows, but here by in-plane flows. We bring this out in a semi-quantitative way in the final part of the paper, where we present observations of the relation between the velocity gradient of the gas flow in a strongly barred galaxy, NGC 1530, and the local formation rate of massive stars, inferring that compression enhances the local SFR, while shear reduces it. 


\section{Direct and indirect evidence for continuous gaseous inflow to the Galactic disc}

Long before galaxy mergers were considered important, Larson (1972a,b) suggested that long term infall of low metallicity gas to the Galactic disc could explain the observed relative dearth of metal poor stars (the G-dwarf problem). An advantage of this scenario over its rivals is that it offers an explanation for the presence of measurable deuterium abundance in the Galaxy, notably near the centre (Audouze et al. 1976, and more recently Lubowich et al. 2000). With no known source of D within the Galaxy, and as astration destroys it, continuous replenishment by infall can explain its presence. High redshift D abundances from the Ly $\alpha$ forest (e.g. Kirkman et al. 2000; Pettini \& Bowen 2001) are $\sim 2-4 \times 10^{-5}$, while recent FUSE values for the local ISM (e.g. Lehner et al. 2002; Oliveira et al. 2003) are $\sim 1-2 \times 10^{-5}$, and to complete the argument for infall the D abundance recently measured with FUSE in the high velocity cloud (HVC) "complex C" (Sembach et al. 2004) is $2.2 \times 10^{-5}$, a value intermediate between the first two. The idea is that some HVC's are supplying metal-poor non-astrated gas continuously to the Galaxy: they are the infall component mentioned. Some early models of Galactic inflow (Hunt \& Sciama, 1972; Tinsley 1977), which derived infall mass rates similar to the Galaxy-wide SFR, entailed a sweeping up of Local Group gas, while Larson (1976) suggested collapsing remnants of the pregalactic nebula. Muller, Oort \& Raimond (1963) detected HVC's in H I at $21 \mathrm{~cm}$; Oort (1970) noted that some of them have radial velocities higher than the local Galactic escape velocity, and might be primordial. Recent observations that their metallicities are $\sim 0.1$ solar (e.g. Lehner et al. 2002; Wakker et al. 2003; Savage et al. 2003; Sembach et al. 2004), rule out primordiality, but also rule out a Galactic origin, so they are candidates for low metallicity infall. Evidence that some HVC's may well belong to the local group but not the Galaxy had been accumulating (Mirabel 1982; Lepine \& Duvert 1994; Blitz et al. 1999; LópezCorredoira et al. 1999; Casuso \& Beckman 2001; Gibson et al. 2001; Putman et al. 2002; and recently Braun et al. 2002) in a sensitive H I survey, estimated

that a gas mass of a few times $10^{9} \mathrm{M}_{\odot}$ could have accreted to both the Milky Way and M31 during their lifetimes. Direct evidence for infall for other galaxies is small. Phookun et al. (1993) detected H I falling onto NGC 5254, while recently Fraternali et al. (2004) detected flows perpendicular to the planes of nearby spirals using $\mathrm{H} \alpha$ as the tracer. Observations of infall to external galaxies are few so far because the detection sensitivity has been limited at $\sim 10^{8} \mathrm{M}_{\odot}$ of gas, while it is probable that the bulk of these flows are in clouds of lower mass. As sensitivities have improved, signs of accretion are being reported, see e.g. van der Hulst \& Sancisi (2003). 


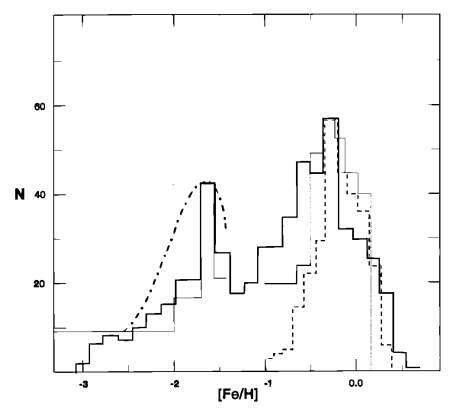

Figure 1. Early frequency histogram of local $\mathrm{G}$ dwarfs as a function of metallicity (using $[\mathrm{Fe} / \mathrm{H}]$ as parameter) from CB97. Two peaks, for halo and thin disc stars, are clearly seen. The thick disc contribution, less obvious, lies between them and widens the higher metallicity peak (for the disc stars).

The G-dwarf problem is a strong pointer to infall for the Milky Way (van den Bergh 1962; Schmidt 1963; Pagel \& Pachett 1975; Tinsley 1980; and Pagel 1987). Closed box models for chemical evolution of the Galactic disc predict far more low metallicity stars than those observed, and integrated population studies of external galaxies point to the same conclusion (Worthey et al. 1996; Espana \& Worthey 2002; Bellazzini et al. 2003). Late F and G stars may be nearly as old as the disc, and preserve a record of its evolution though the oldest have evolved off the main sequence, so as improving technique brought K-dwarf statistics within range (Flynn \& Morrell 1997) their local metallicity distribution has given an more accurate history to follow. Excluding halo and thick disc stars kinematically, a frequency $v$. metallicity plot for $\mathrm{G}$, or better $\mathrm{K}$ dwarfs show low numbers for low disc metallicity $([\mathrm{Fe} / \mathrm{H}]<-1)$ rising sharply to a narrow peak between $[\mathrm{Fe} / \mathrm{H}]=-0.6$ and -0.2 , then falling off to higher metallicities. The earlier G dwarf statistics (Carney et al. 1990; RochaPinto \& Maciel 1996) are well explained in a scenario with near constant infall of low metallicity gas to the Galactic plane (Casuso \& Beckman 1997). The K dwarfs show the same metallicity pattern (Flynn \& Morrell 1997; Favata et al. 1997; Kotoneva et al. 2002) and we will discuss this and how it is modelled in Sect. 4 below. Fig. 1 shows an early stellar metallicity-frequency plot, taking in thin disc, thick disc and halo G-dwarfs from Casuso \& Beckman (1997) (hereinafter CB97).

Further chemical evidence explained well by low metallicity infall is the evolution of beryllium and boron, measured $v$. iron as standard in local disc stars. A recent compilation of observational data on this is shown in Fig. 2 (left and right). The "loop-back" seen for disc stars in both plots is well explained by infall, and best explained if the infall rises slowly with time. Recent models for Be and B evolution in the Galaxy (Fields et al 2000; Ramaty et al. 2000) have focused on their evolution in the halo, i.e. for $[\mathrm{Fe} / \mathrm{H}]<-1$, 

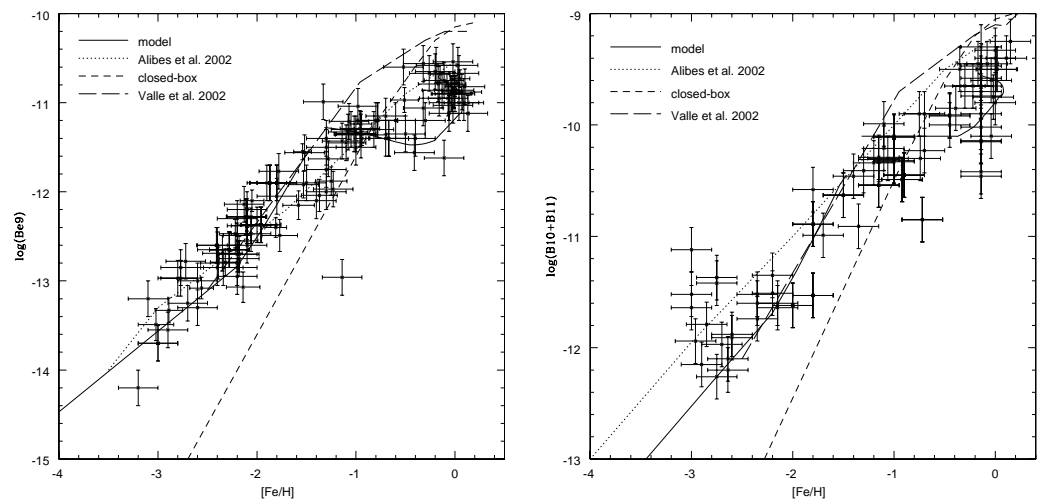

Figure 2. (left) Compilation of Be abundances measured in local disc and halo stars (taken from CB04). Model predictions shown are for a closed box model, for two models with infalling $\mathrm{H}$ I but at rates which decline with time (Alibés et al. 2002; Valle et al. 2002) and one model, for the disc, with slowly rising infall: CB97, solid line. For more details see text. (right) Compilation of B abundances measured in local disc and halo stars, taken from CB04. Model predictions are from the same sources as in Fig. 2 (left).

invoking specific mechanisms for their production by high cosmic ray fluxes in star forming zones to explain the observed linear relations of both $\mathrm{Be}$ and $\mathrm{B}$ with Fe. But their treatment of the disc evolution of Be and B lacks finesse, notably in the chemical evolution model used, mainly because the authors are not really aiming to explain the disc observations, even though over $90 \%$ of $\mathrm{Be}$ and B were made in the disc. Alibés et al. (2002) use a much better disc model, but even they do not predict very well the observations in Fig. 2. In CB97 we had managed to model earlier versions of these data sets. To our surprise, even concern, the model entailed $\mathrm{H}$ I infall with a rising rate over the disc lifetime. In 1997 the B observations were sparse but as shown in Fig. 2 the trends for $\mathrm{B}$ and $\mathrm{Be} v$. Fe have been strongly confirmed as data has accumulated. It was these $\mathrm{B}$ and Be observations which first pushed us towards an increasing inflow rate scenario, but other types of evidence have accumulated to support this as we now describe.

\section{The history of the local star formation rate (SFR)}

Since Vaughan \& Preston (1980) brought out the possible use of chromospheric activity in late type dwarfs as a chronometer, a number of surveys within $1 \mathrm{kpc}$ of the Sun have used activity indices to investigate the time dependence of the local SFR. Barry (1988) found using this technique that the SFR, averaged in bins of $10^{9} \mathrm{yr}$ showed evidence of a secular increase during the disc lifetime, but also large amplitude excursions in this general trend. From a much wider stellar sample Rocha-Pinto et al. (2000) confirmed both 


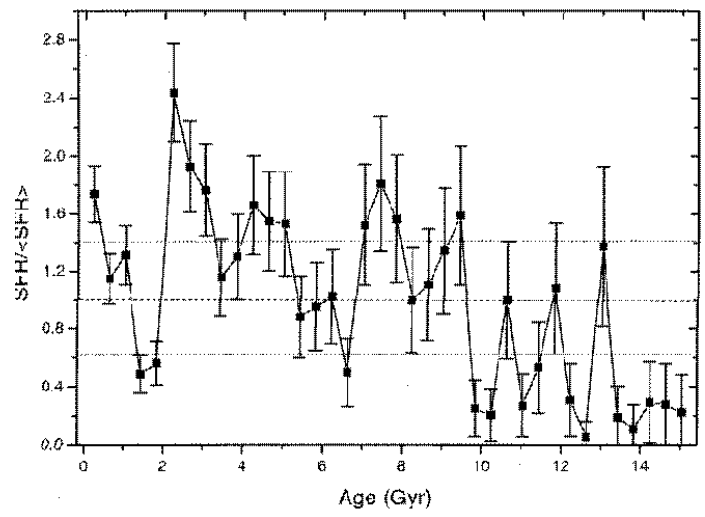

Figure 3. Star formation rate as a function of epoch, from Rocha-Pinto et al. (2000) based on stellar activity, and from observations of stars within $1 \mathrm{kpc}$ of the Sun. Although absolute ages are progressively less accurate as they increase, the general run of SFR has clearly increased with increasing time, on which major shorter term variations are superposed.

findings, as we show here in Fig. 3. Although both samples were taken locally to the Sun, Wielen $(1977,1996)$ showed that the diffusion of stellar orbits implies that such a sample must include stars which have drifted radially from their birthplaces by over $2 \mathrm{kpc}$, both inwards and outwards. Thus any large excursion in the SFR must have occurred simultaneously over a major fraction of the Galactic disc. Interaction could cause this, but not only with another galaxy; accretion of a large gas cloud will also work. The data in Fig. 3 are well interpreted in a scenario of $\mathrm{H}$ I infall of clouds with a range of sizes. We can estimate a time scale for single cloud accretion, rough as it depends on distance estimates, using measured properties of extragalactic HVC's. From Blitz et al. (1999) or Putman et al. (2002) we set a lowish scale size for a large cloud at $\sim 5 \mathrm{kpc}$. Measured radial velocity of $\sim 200 \mathrm{~km} \mathrm{~s}^{-1}$ then gives an accretion time of a few times $10^{8} \mathrm{yr}$, which is consistent with the widths of the surge peaks in Fig. 3. Also from Fig. 3 we can see a long term slowly rising trend, which is clearly worth further comment.

Is this apparently rising SFR compatible with cosmological trends? Since the Madau et al. (1996) plot it is known that the global SFR in the universe has been falling steadily from $z=1$ to the present epoch. We must note though that the Local Group is gravitationally bound; interaction rates between galaxies have been steady since the group condensed. The HVC accretion rate to the Milky Way must depend on the density of the clouds within the Local Group, the gravitational range of the Galaxy, and its accretion cross-section. While accretion causes the first parameter to fall with time, it causes the second and third to rise as gas accumulates in the plane. In López-Corredoira et al. (1999) we computed that for the current accretion rate to be rising (or constant in the 
limiting case) the present mean cloud density must have a lower limit, which can be converted to an estimate of the fractional mass of the Local Group in the form of these intracluster clouds. The value is $\sim 50 \%$, which is fully plausible, comparing the masses of its galaxies (the Milky Way and M31 as the smaller objects contribute little) with dynamical estimates of the Local Group mass, as first done by Kahn \& Woltjer (1959); for a recent estimate see Whiting (1999). (Note that these conclusions depend on only one assumption about dark matter: that the baryon to dark matter density does not vary much from object to object, which we cannot discuss in detail here, but we take as reasonable). We have also shown, in López-Corredoira et al. (2002) that if the net long term vector of this inflow is directed to the Local Group barycentre (Blitz et al. 1999) its dynamic parameters are those required to yield the known Milky Way H I warp amplitude and direction. A global check on the inflow rate can come from measurements of the SFR. For example SN II rates, of order a few per century in the disc, (e.g. Dragicevich et al. 1999) agree with an accretion rate of a few $\mathrm{M}_{\odot} \mathrm{yr}^{-1}$, using a Salpeter IMF with mass limits $0.1 \mathrm{M}_{\odot}$ and $100 \mathrm{M}_{\odot}$, and that type II SNe come from stars with $\mathrm{M}>8 \mathrm{M}_{\odot}$. Chemical evolution models require mean accretion rates during the disc lifetime of $\sim 2 \mathrm{M}_{\odot} \mathrm{yr}^{-1}$, consistent with estimates of HVC accretion rates (Blitz et al. 1999; Braun et al. 2002).

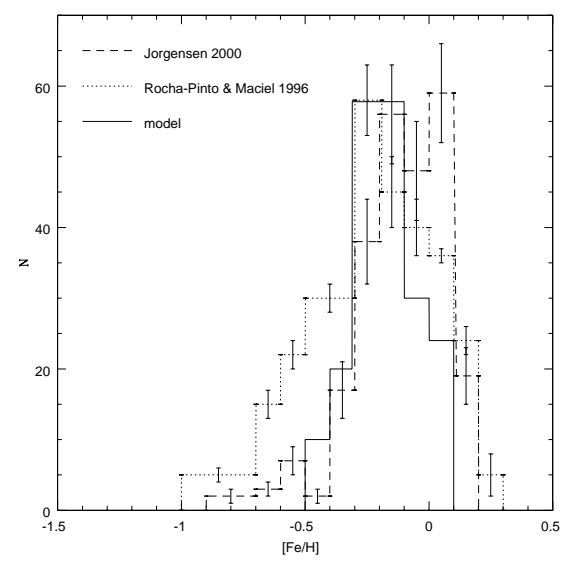

Figure 4. Frequency histograms of metallicity for $\mathrm{G}$ dwarfs in the local Galactic disc, from Rocha-Pinto \& Maciel (1996), and from Jorgensen (2001). In the latter a scale height criterion has been used to minimize the thick disc contribution. Compared with these data is a model based on chemical evolution with increasing $\mathrm{H}$ I infall from CB04.

\section{The K-dwarf problem}

The dearth of low metallicity $\mathrm{K}$ dwarfs in the Galactic disc is a more severe test of chemical evolution models than previous similar data for $\mathrm{G}$ dwarfs. In a recent article (Casuso \& Beckman 2004) we describe the observational and the- 
oretical developments in this field and this section gives an overview of these. The G-dwarf data by Rocha-Pinto \& Maciel (1996) already showed a much narrower peak in the metallicity frequency distribution than that in Fig. 1. Using a chemical evolution model (CB97) developed to explain the Be and B data, in Casuso \& Beckman (2001) (CB01) we modelled this narrow peak well. The model included a rising infall rate, and predicted even better the $\mathrm{G}-\mathrm{dwarf}$ data of Jorgensen (2001), published while CB01 was submitted, so this was a genuine prediction and not a post-diction as one so ofter finds. It is significant that we selected the best model for its Be, B predictions, although improvements were possible using only the Rocha-Pinto \& Maciel (1996) data, so the improved agreement with Jorgensen was remarkable. One feature of Jorgensen's data was the careful exclusion of thick disc stars from the sample. In Fig. 4 we compare the two data sets with the model from CB01; these fits,especially to Jorgensen's data, strengthened our "increasing infall rate" hypothesis. The next step was to use K-dwarf metallicity distributions. In Fig.5 we show two data sets, by Favata et al. (1997) and Kotoneva et al. (2002), compared with our model prediction. We can see that agreement is good in the metallicity range close to solar, but less good outside this range. Of the two data sets, one lies above and the other below our prediction, at lower metallicities. The cause in both cases is the thick disc population. Favata et al. (1997) cut off their graph where the thick disc numbers become appreciable, while Kotoneva et al. (2002) did not take special steps to exclude thick disc stars. We conclude that the $\mathrm{K}$-dwarf metallicity distribution in the thin disc gives some support to our model, but further measurement and analysis are needed here.

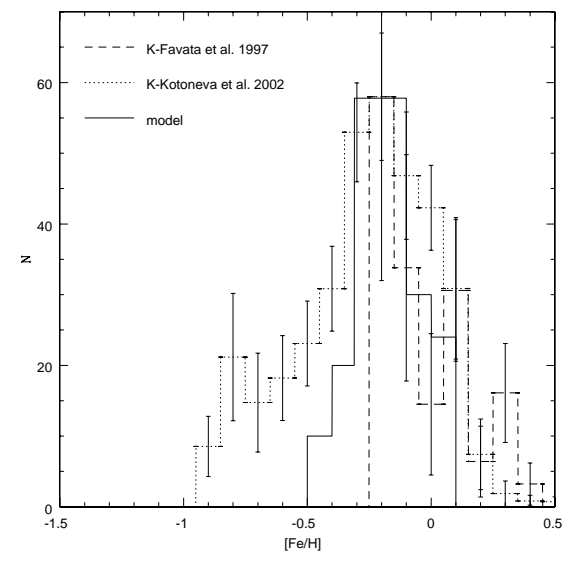

Figure 5. Frequency histograms of metallicity for $\mathrm{K}$ dwarfs in the local Galactic disc, from Favata et al. (1997) and Kotoneva et al. (2002). Compared with these data we show a model based on chemical evolution with increasing $\mathrm{H}$ I infall from CB04. Agreement is not as good as for the G-dwarfs in Fig. 4, but no attempt to limit the sample to thin disc stars was made in either observational paper, while the model is strictly for thin disc evolution. 


\section{The light element abundances in the Galactic Disc}

The light elements: hydrogen, helium, lithium, beryllium and boron, are exceptional in that their main production sites are principally non-stellar. Hydrogen $(\mathrm{H}, \mathrm{D}$ and $\mathrm{T})$ most present day helium $\left({ }^{4} \mathrm{He}\right.$ and $\left.{ }^{3} \mathrm{He}\right)$, and some $10 \%$ of lithium are primordial, while the remaining lithium, beryllium and boron were formed in the interstellar medium (ISM) in processes involving Galactic Cosmic Rays (GCR). Reeves (1974) reviewed light element nucleosynthesis setting the scene for all subsequent developments. For the present article the importance of the light elements is that they present sensitive tests of abundance dilution by infall of element-poor gas into the ISM where they are being produced. We have presented the Be and B $v$. Fe data plots in Fig. 2, and in Fig. 6 we show a similar compilation for Li. For Be and B there has been much interest in why they show linear dependences on metallicity; they are spallation products of CNO so initial predictions were for a quadratic dependence, as secondary elements. Without entering in depth in this complex field, it is clear from Fig. 2 that this linear relation holds only in the halo metallicity range $([\mathrm{Fe} / \mathrm{H}]<-1.5)$ while for disc metallicities the dependence is far from linear. Clearly halo and disc behaviour are different, and this can be explained independently of the specific GCR production mechanisms for Be and B by differences in the surrounding gas flow conditions. For the halo stars, not the subject of this article, a model in which the star forming gas is flowing inwards to the Galactic centre as the initial spherical collapse occurs, gives a satisfactory explanation of the pseudo linear Be and $\mathrm{B} v$. Fe observations. For the disc stars we show four model comparisons, a closed box model and three inflow models. The best fits are for secularly slowly increasing inflow. The same model, with no fine tuning, was used to fit the G- and K-dwarf distributions shown in Figs. 4 and 5, and is used as a framework for predicting the $\mathrm{Li} v$. Fe plot in Fig. 6, though here the importance of both production and destruction mechanisms is such that the goodness of fit is not a simple test of the gas flow model (see Casuso \& Beckman 2000 for more details). Finally we can return to the case of deuterium which we discussed briefly above. As deuterium is destroyed in stars, there are no equivalent plots to those in Fig. 2 for this nuclide. However the mere presence of D in the ISM of the Galaxy today, especially near the Galactic centre, implies very recent infall, and we discussed implications of this in Casuso \& Beckman (1999). To summarize this section, the best fits to the observed evolutionary plots of light element abundances in the Galactic disc are provided by models in which the infall of $\mathrm{H}$ I has occurred at a constant or slowly rising rate. 


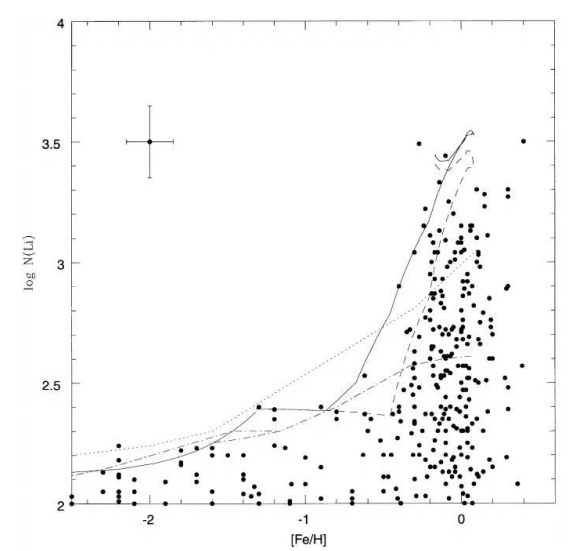

Figure 6. Lithium abundance as a function of metallicity $([\mathrm{Fe} / \mathrm{H}])$ for local disc stars; compilation from Casuso \& Beckman (2000). The upper envelope represents minimum Li depletion in the star observed, and is the datum for model fitting. Compared are a number of models, also from Casuso \& Beckman (2000). The complexity of the production and destruction processes implies that model fits do not tell us too much about the time dependence of gaseous infall for $\mathrm{Li}$, but all models showing fair fits to the upper envelope use constant or increasing infall assumptions.

\section{Gas accretion in the general process of galaxy evolution}

Far less attention has been paid to gas accretion as a driver of galaxy evolution than to mergers, because the latter are more spectacular and much easier to observe. The lower end of the galaxy mass function should contain clouds whose baryonic mass could be as high as $10^{8} \mathrm{M}_{\odot}$, and would range downwards from there. Their numbers should be high, conforming to general laws of mass fractionation, which Elmegreen (2002) has characterized as fractal in his discussion of Galactic gas clouds. Estimates at the low end of the galaxy mass function by Bell et al. (2003) confirm that the number of objects is rising as the gas to stars ratio is rising, for the faintest dwarf galaxies, and should keep rising for "failed galaxies" which are the gas clouds we "need". Rosenberg \& Schneider (2002) find a slope of -1.5 for the H I mass function for galaxies. $\lambda$ CDM model cosmologies (see Kauffmann, White \& Guiderdoni 1993, for an early model including $\lambda$ ) do predict a steep mass function at the low mass end, though they also overpredict satellite galaxy numbers, which is a problem. So we would expect galaxy groups and clusters to contain clouds in the mass range proposed here, but most such objects lie below present $\mathrm{H}$ I detectability limits $\left(\sim 10^{7} \mathrm{M}_{\odot}\right)$ and can be picked up only in nearby galaxy groups.

One result of considerable relevance is that by Kennicutt, Tamblyn \& Congdon (1994). In a survey they found that early type spirals have much lower current SFRs than late types (as expected) but a much higher fractional variability in the SFR. Tomita et al. (1996) inferred that their results imply SFR 
variability on timescales of $10^{8}$ years, and Hirashita \& Kamaya (2000) modelled this using a scenario with quasi-periodic limit cycles. One problem with this mechanism is to explain how SFR surges can occur over a whole galaxy. We have shown how the SFR excursions in Rocha-Pinto et al. (2000) imply Galaxy-wide changes, which are consistent with triggering by external $\mathrm{H}$ I clouds if these are large enough to affect a large part of the Galaxy: sizes of several kiloparsecs for the "external" HVC's were estimated by Blitz et al. (1999) and Putman et. al (2002). The variability difference between early and late type galaxies noted above is because a gas rich disc presents a larger cross-section for cloud accretion, and a higher probability for a star formation surge when a cloud is captured. Late types respond only to the most massive incoming clouds, and do so in a less dramatic way.

The current gas accretion rate of $2 \mathrm{M}_{\odot} \mathrm{yr}^{-1}$ cited in Sect. 3, assuming constant infall, would imply $2 \times 10^{10} \mathrm{M}_{\odot}$ of gas accreted in the disc lifetime; an exponentially rising rate starting from zero would give $1.4 \times 10^{10} \mathrm{M}_{\odot}$. Using a dark to baryonic matter ratio of 5 the total mass accreted would be between 7 and 10 times $10^{10} \mathrm{M}_{\odot}$. These total masses will be upper limits as the dark matter component of any HVC with incoming velocity higher than escape velocity will not be retained (this would give a natural explanation for possible low dark matter: baryonic matter ratios in massive late type spirals). These predictions are just compatible with observationally based estimates. Nikiforov et al. (2000) estimate a Galaxy disc mass of $6 \times 10^{10} \mathrm{M}_{\odot}$ within a $20.5 \mathrm{kpc}$ radius, in a total Galaxy mass of $3.3 \times 10^{11} \mathrm{M}_{\odot}$. Even if these figures are rough, we find that a major fraction of the disc has been accreted by gas infall. This is qualitatively compatible with "inside-out" evolution of the disc, in which growing gas column density as a function of time and radius have produced increased SFRs at smaller radii, which in turn yields a radial metallicity gradient in the disc. Inside-out disc formation occurs in many $\lambda$ CDM based scenarios (e.g. Samland \& Gerhard 2003). In any model where the disc is built up by gas accretion the column density threshold for quiescent star formation will give higher SFR's in the inner disc, although diffusion, especially if impelled by a bar, will tend to blur out the radial distribution of stellar age and metallicity. As it is extremely hard, as yet to estimate stellar population age distributions from outside a galaxy, the local measurements we have used to predict the time dependence of infall must be used as the best benchmark for some time to come.

\section{And now for something completely different: how gas flows affect star formation in a barred galaxy}

\subsection{Non-circular velocities around the bar}

So far we have been discussing accretion inflow perpendicular to the planes of galaxies. In this shorter final section we will see how gas flows within the 

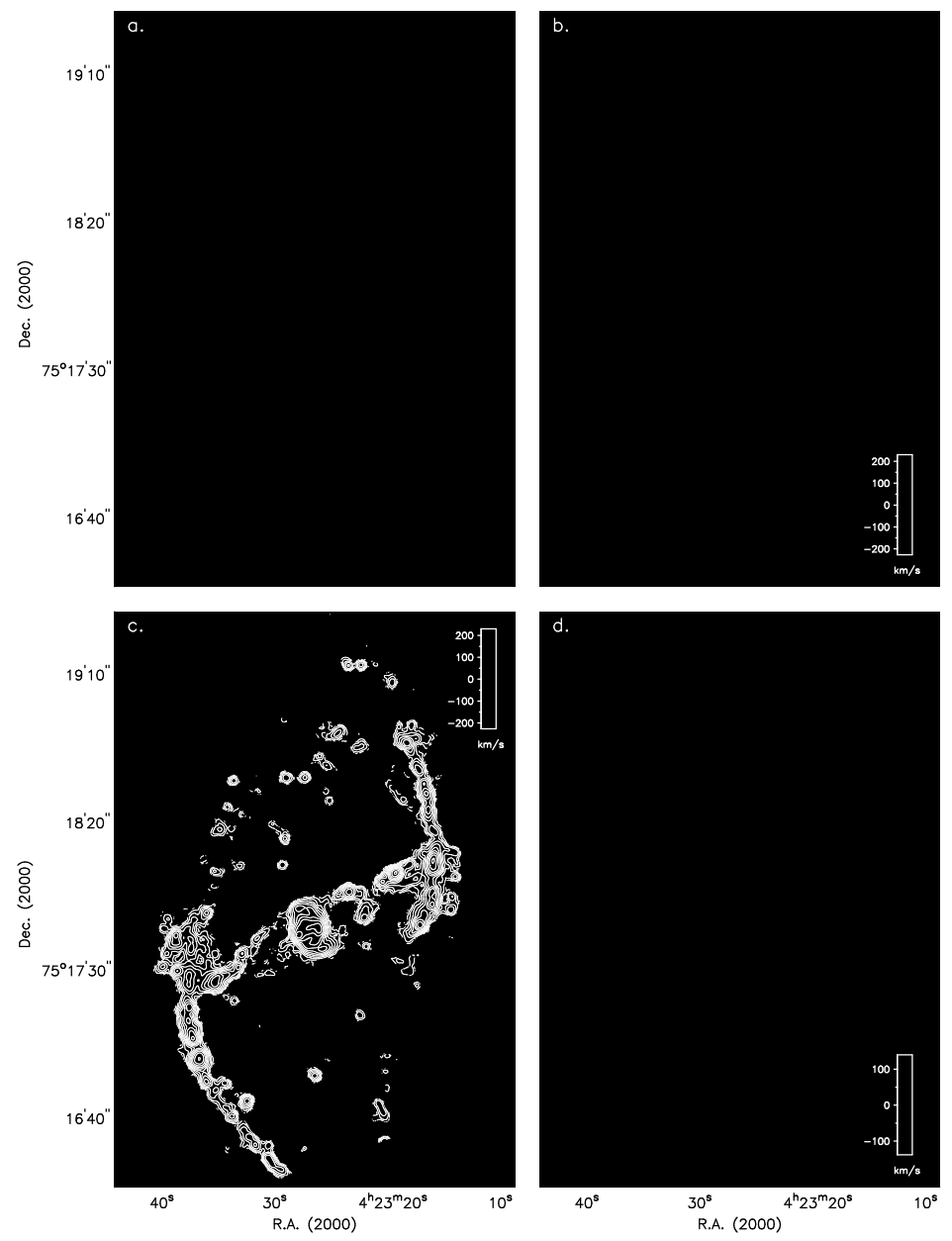

Figure 7. (a) Surface brightness map in H $\alpha$ of NGC 1530, from a TAURUS data cube from the $4.2 \mathrm{~m}$ WHT La Palma. (b) Radial velocity map of ionized gas from the peaks of the $\mathrm{H} \alpha$ emission lines across the face of the galaxy, using the same data cube. (c) Contours of H $\alpha$ surface brightness superposed on a two-dimensional projection of the rotation curve derived from the velocity field shown in (b). (d) Map of the residual, non-circular velocity field, obtained by subtracting off the projected rotation curve in (c) from the complete velocity field in (b). The strong non-circular velocity field aligned with the bar is clearly seen here, as the galaxy inclination causes the flow along one side to be directed towards us, and away from us along the other side of the bar (see text for more details, also see Zurita et al. 2004).

plane affect the local SFR on short timescales. For this we use our recently measured velocity field of the strongly barred galaxy NGC 1530. In Zurita et al. (2004) we describe the TAURUS Fabry-Pérot interferometer on the $4.2 \mathrm{~m}$ 
WHT, La Palma (Spain) with which we made the two dimensional velocity map of the ionized hydrogen using $\mathrm{H} \alpha$ emission, and give details of the observations and reduction. The map has a velocity interval of $\sim 18.6 \mathrm{~km} \mathrm{~s}^{-1}$ and an angular resolution of $\sim 1^{\prime \prime}$, which mark improvements on previous optical and radio maps by Regan et al. (1996), by Downes et al. (1996) and by Reynaud \& Downes $(1997,1998,1999)$. We derived first the classical rotation curve, and used its two dimensional projection to subtract off from the observed velocity field, yielding a residual field of non-circular projected velocity over the full face of the galaxy. In Fig. 7 we show how this was done and illustrate our results. NGC 1530 is an excellent subject for kinematic analysis. Its arms, bar and circumnuclear disc are very well separated; the major axis and bar position angle are well separated, and the inclination permits good velocity and intensity measurement. In Fig. 7c, streaming in the arms due to the disc density wave system shows up as ripples in the two dimensional velocity field, with amplitude 20-30 $\mathrm{km} \mathrm{s}^{-1}$, which we will not analyze further here. In Fig. 7b the non-circular motions round the bar show up clearly as distorted isovels. These motions are well seen in Fig. 7d: the residual non-circular velocity field, after subtracting off the rotational field seen in Fig. 7c. Cross-sections through this field are shown in Fig. 8, revealing counterflow with amplitude $\gtrsim \pm 100$ $\mathrm{km} \mathrm{s}^{-1}$ top either side of the bar.
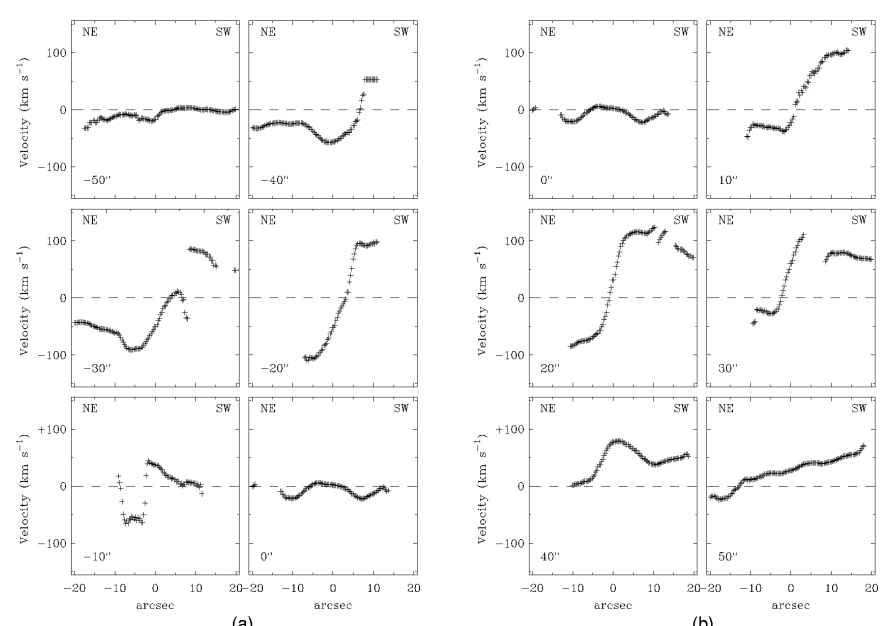

(b)

Figure 8. Cross-sections perpendicular to the bar of the non-circular velocity field illustrated in Fig. 7d, showing the amplitude of the flows, $>100 \mathrm{~km} \mathrm{~s}^{-1}$ in opposite directions at either side of the bar.

Although $\mathrm{H} \alpha$ gives patchy velocity fields compared with $\mathrm{H}$ I, we have high spatial resolution to compensate. This allows us to produce maps of noncircular velocity gradient across the whole galaxy from a few hours' observa- 

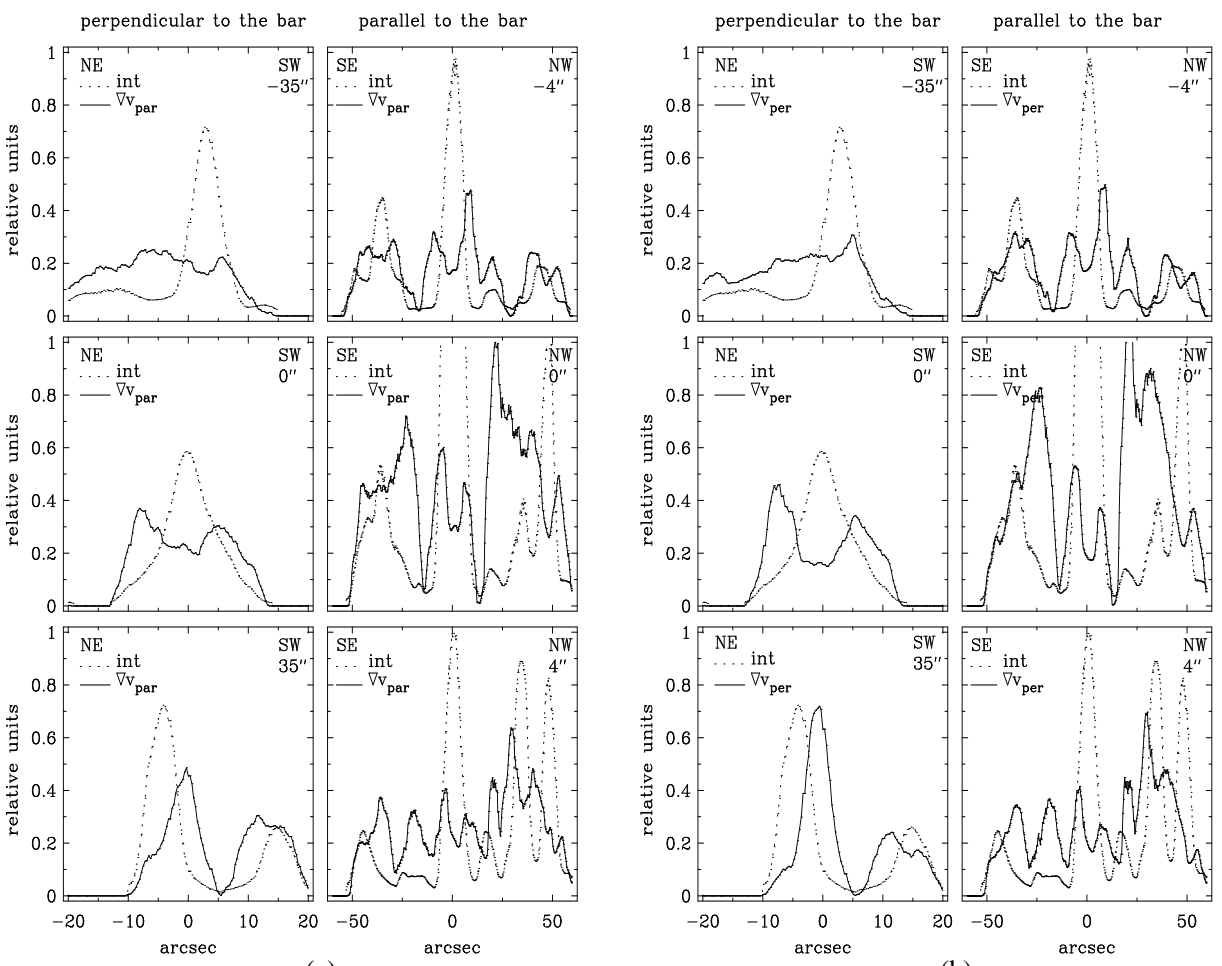

(a)

(b)

Figure 9. (a) Cross-sections, in the directions indicated, of the surface brightness in $\mathrm{H} \alpha$ and of the gradient in velocity parallel to the bar. (b) Cross-sections in the directions indicated, of the surface brightness in $\mathrm{H} \alpha$ and of the gradient in velocity perpendicular to the bar. In both graphs we clearly see local maxima in brightness (SFR) at local minima in velocity gradient. For further explanation see text, also Zurita et al. (2004).

tions. In a poster complementary to this article (Zurita et al. 2004a) we show a beautiful result of this work: the complete coincidence of the strong dust lanes along the bar with the loci of maximum velocity gradient perpendicular to the bar. This shows that lines of maximum shear, which are also lines of net zero non-circular velocity, are zones to which the dust migrates. We can draw more powerful inferences by superposing cross-sections of our maps in velocity gradient on cross-sections across the surface brightness map (Fig. 7a); the results are shown in Fig. 9. These show clearly an anticorrelation between local SFR (H $\alpha$ surface brightness) and local velocity gradient,but in two distinct regimes. In zones of high shear (gradient perpendicular to the flow along the bar) star formation is suppressed, a conclusion inferred by Reynaud \& Downes (1998) from CO mapping. This is as expected if shear disrupts large molecular clouds on timescales shorter than those for massive star formation (see Kennicutt 1998 for a discussion of this and related points). In zones of high compression star formation is enhanced, but occurs offset by hundreds of parsecs from the zone 
of maximum present compression. The offset relation; compression-high local SFR is seen on scales of hundreds of parsecs. On smaller scales we see the effects of outflow from luminous $\mathrm{H}$ II regions which impinges on the surrounding ISM, yielding "walls" of velocity gradient around these regions. These can be seen in Fig. 1 of Zurita et al. (2004a), and show up here in Fig. 9, where each peak in the $\mathrm{H} \alpha$ surface brightness due to a bright $\mathrm{H}$ II region coincides with a dip surrounded by symmetrically offset peaks, in the velocity gradient. These outflows have been reported by Relaño et al. (2003), and have amplitudes of $40-80 \mathrm{~km} \mathrm{~s}^{-1}$ from the centre of the $\mathrm{H}$ II region. We will present a quantitative treatment of these outflows and some initial models in Relaño et al. (2004).

\subsection{Gas flow spiralling to the nucleus in the inner disc}

In the inner $5^{\prime \prime}$ of our original rotation curve (Zurita et al. 2004) we found a steep gradient which relaxed to a slightly less steep gradient outside this radius. Assuming this to be caused by a bulge, we used an HST-NICMOS near-IR image to check this, but as seen in Fig. 10a the central spheroidal component is diminutive, and could not cause the steep inner gradient. Assuming that we are detecting projected non-circular motion, we extrapolated the outer part of the rising rotation curve linearly to the origin, and subtracted this from the observed inner velocity field, yielding a map of projected non-circular velocity in the inner disc, as shown in Fig. 10c. This "yin/yang" pattern reveals spiral inflow to the nucleus. To show this we took an image of the zone in $\mathrm{J}-\mathrm{K}$ from Pérez-Ramírez et al. (2000), see Fig. 10b, which shows interlocking spiral dust lanes. Assuming that these indicate inward flow lines we computed the projected velocity we would see, taking for simplicity a constant velocity amplitude along the flow. The result, in Fig. 10d agrees nicely with the observational field in Fig. 10c, except at the north and south ends of the inner disc, where we know that the $\mathrm{H} \alpha$ data do not allow us to plot the true circular rotation curve, so the residual map in Fig. 10c will have systematic errors here. This inward spiralling flow is not due to an inner bar. In Zurita et al. (2004, 2004a) we show using unsharp masking of the HST image that any circumnuclear bar must be less than $0.5^{\prime \prime}$ in length, and with negligible dynamical effect over the inner disc. However, as modelled by Englmeier \& Shlosman (2000) in a galaxy with an inner Lindblad resonance at a radius within the length of a major bar, as the dominant stellar $x_{1}$ orbits in the bar give way to perpendicular $x_{2}$ orbits within the ILR the gas is pulled round into opposing spiral trajectories, very much as seen in Fig. 10, which take it down to within a couple of hundred parsecs of the nucleus. Fabry-Pérot velocity fields from ionized gas emission give us an excellent tool for exploring these dynamical effects. 
16

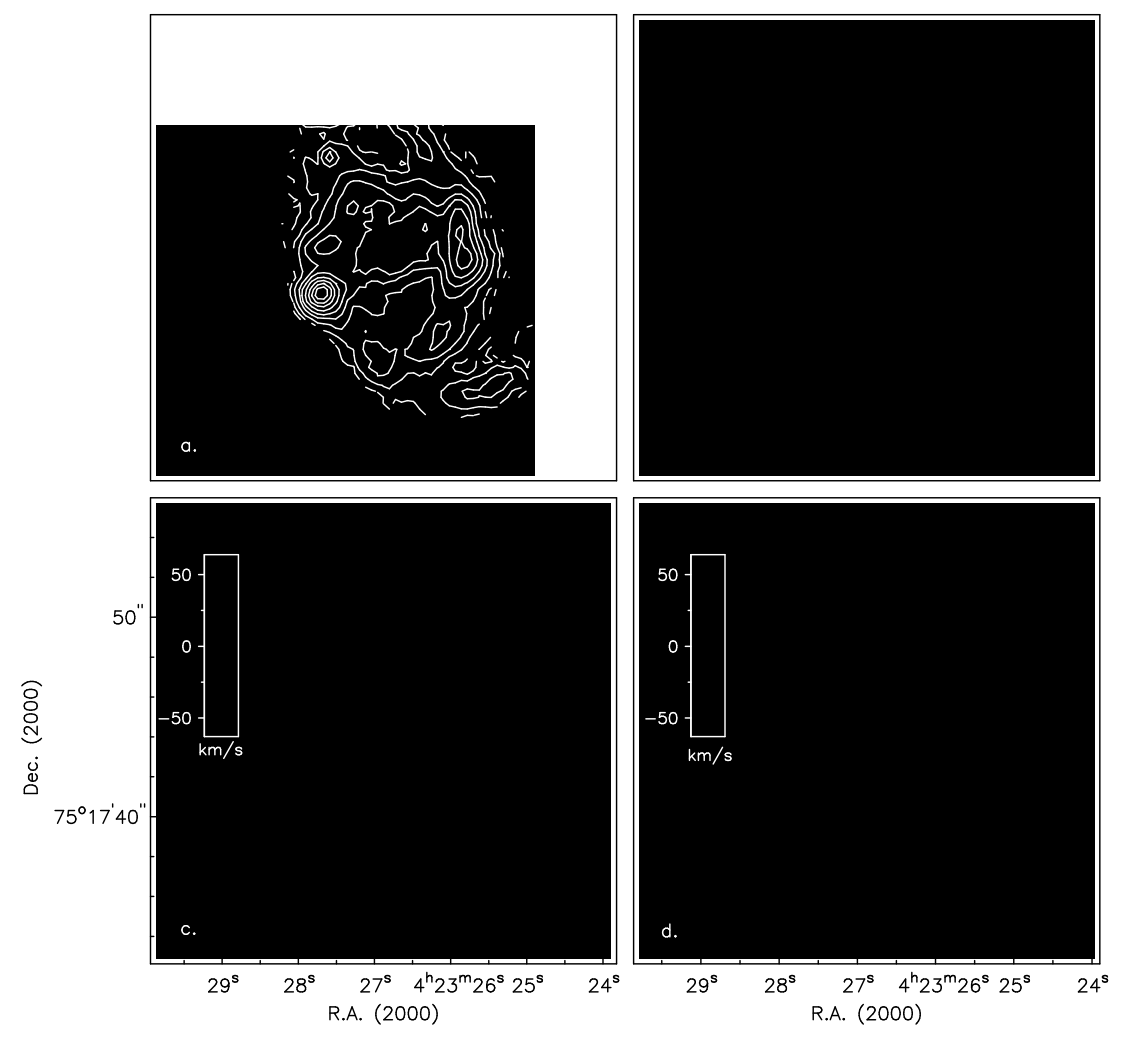

Figure 10. (a) HST-NICMOS H band image of the central disc of NGC 1530, with $\mathrm{H} \alpha$ contours superposed. (b) J-K image of the zone, from Pérez-Ramírez et al. (2000), clearly showing the interlocking spiral dust lanes around the nucleus. (c) Residual non-circular velocity map, projected along the line of sight, for the circumnuclear disc of NGC 1530, obtained from Fabry-Pérot observations. (d) Model predicted velocity field, based on constant amplitude flow vectors directed along the paths defined by the dust lanes in (b). The predictions match very well the observed pattern, notably within 5 " of nucleus, and show the power of the Fabry-Pérot method for velocity field diagnostics.

\section{Some general conclusions}

The message of this article is the same for the two different astrophysical systems considered: galaxy evolution cannot be well understood without a knowledge of gas flows and their effects on star formation. For the Milky Way our reasoning has been indirect. Chemical evidence is used to show that gas has been accreting to the disc during its whole lifetime, and at rates which do not appear to have declined during this period. This does not contradict the general picture in which the SFR in the universe has been declining steadily from $z=1$ to the present epoch, as the overall density has fallen. We note that the ongoing global disc SFR is of order $2 \mathrm{M}_{\odot} \mathrm{yr}^{-1}$, while during the formation 
of the initial spherical component (stellar halo) the rate must have been much higher. This means that a constant, even slowly increasing SFR in the discs of galaxies and small groups, regulated by inflow and feedback processes, need not contradict inferences about the universal SFR from the Madau et al. (1996) plot and its subsequent refinements. One feature of star formation in galactic discs is its apparently low efficiency averaged over time. In a model where the disc mass has grown slowly via accretion, this low efficiency is only apparent, an effect of time averaging. Any gas which arrives as infalling $\mathrm{H}$ I suffers relatively rapid conversion to stars, subject to feedback from the massive stars themselves. This scenario of gas persistently raining down onto galaxy discs, with the occasional sharper shower, a more massive $\mathrm{H}$ I cloud, is finding support as $\mathrm{HI}$ detection sensitivities increase, and more $\mathrm{H}$ I clouds falling in to other galaxies are detected. And infall can explain rather well what other mechanisms (major interactions apart) cannot: how the star formation rate can increase and decrease simultaneously over the whole, or the major part of a Milky Way sized disc galaxy. We have considered here the evidence that this does happen, both for the Milky Way and for galaxies in general.

In the second part of the paper we have shown how gas flow is a key parameter determining the star forming pattern in NGC 1530. While shocks, strong velocity gradients along the line of flow, yield massive stars, shear, strong velocity gradients perpendicular to the flow, inhibit massive star production. NGC 1530 is a specially favourable case for study, for the essentially geometrical and morphological reasons explained in Sect. 7, but we should treat it here as an example revealing processes of general importance. Here we have been able to present a semi-quantitative picture, but to disentangle fully the effects produced by gas flow on star formation we will need, as observers, to combine similar information from ionized, neutral (atomic) and molecular gas. Only then will we have the basis for full quantitative understanding, aimed at the major prize: a physical theory of star formation in different galactic environments.

The research discussed in this article was supported by grants AYA2001-0435 (Spanish Ministry of Science and Technology) and AYA2004-08251-C02-01 (Spanish Ministry of Education and Science). A. Zurita acknowledges support by the Consejería de Educación y Ciencia de la Junta de Andalucía, Spain. The authors thank the organizers of the conference for the invitation to present this paper in an excellent context, both scientific and social.

\section{References}

Abraham, R. G. 1999 in "Galaxy Interactions at Low and High Redshifts, (Eds. J.E. Barnes and D.B. Sanders), Dordrecht, Kluwer, p.11

Alibés, A., Labay, J., \& Canal, R. 2002, ApJ, 571, 326

Audouze, J., Lequeux, J., Reeeves, H., \& Vigroux, L. 1976, ApJ, 208, L51

Barry, D. C. 1988, ApJ, 324, 436 
Bell, E. F., McIntosh, D. H., Katz, N., \& Weinberg M. D. 2003, ApJL, 585, 117

Bellazzini, M., Cacciari, C., Federici, L., Fusi Pecci, F., \& Rich, M. 2003, A\&A , 405, 867

Blitz, L., Spergel, D. N., Teuben, P. J., Hartmann, D., \& Burton, W.B. 1999, ApJ, 514, 818

Braun, R., de Heij, V., \& Burton, W. B. 2002, BAAS, 200, 3304

Carney, B. W., Latham, D. W., \& Laird, J. B. 1990, AJ, 99, 572

Casuso, E., \& Beckman, J. E. 1997, ApJ, 475, 155 (CB97)

Casuso, E., \& Beckman, J. E. 1999, AJ, 118, 1907

Casuso, E., \& Beckman, J. E. 2000, PASP, 112, 942

Casuso, E., \& Beckman, J. E. 2001, ApJ, 557, 681 (CB01)

Casuso, E., \& Beckman, J. E. 2004, A\&A, 419, 181 (CB04).

Downes, D., Reynaud, D., Solomon P. M., \& Radford, S.J.E. 1996, ApJ, 461, 186

Dragicevich, P. M., Blair D. G., \& Burman, R. R. 1999, MNRAS, 302, 693

Elmegreen, B. G. 2002, ApJ, 564, 773

Englmeier, P., \& Shlosman I. 2000, ApJ, 528, 677

Espana, L., \& Worthey, G. 2002, AAS, 201, 1408

Favata, F., Micela, G., \& Sciortino, S. 1997, A\&A, 323, 809

Fields, B. D., Olive, K. A., Vangioni-Flam, E., \& Cassé, M. 2000, ApJ, 540, 930

Flynn, C ., \& Morell, O. 1997, MNRAS, 286, 617

Fraternali, F., Osterloo, T., \& Sancisi, R. 2004, A\&A (in press)

Freeman, K. C. 1993 in "Galaxy Evolution: the Milky Way perspective”(Ed. S. R. Majewski) ASP Converence Series, Vol. 49, ASP, San Francisco, 1993

Gibson, B. K., Penton, S. V., Giroux, M. L., Stocke, J. T., Shull, J. M., \& Tumlinson, J. 2001, AJ, 122, 3280

Gilmore, G., \& Feltzing S. 2000 in "The Evolution of Galaxies on Cosmological Timescales" (Eds. J. E. Beckman \& T. J. Mahoney), ASP Conference Series, Vol. 187, ASP, San Francisco, p. 20

Gilmore, G., \& Wyse, R. F. G. 1998, AJ, 116, 748

Hirashita, H., \& Kamaya, H. 2000, AJ, 120, 728

Hunt, R., \& Sciama, D. W. 1972, MNRAS, 157, 335

Jorgensen, B. R. 2001, A\&A, 363, 947

Kahn, F. D., \& Woltjer, L. 1959, ApJ, 130, 70

Kauffman, G., White, S. D. M., \& Guiderdoni, B. 1993, MNRAS, 264, 201

Kennicutt, R. C. 1998, ARA\&A, 36, 189

Kennicutt, R. C., Tamblyn, P., \& Congdon, C. W. 1994, ApJ, 435, 22

Kirkman, D., Tytler, D., Burles, S., Lubin, D., \& O’Meara, J. M. 2000, ApJ, 529, 655

Kotoneva, E., Flynn, C., Chiappini, C., \& Matteucci F., 2002, MNRAS, 336, 879

Larson, R.B. 1972a, Nature, 236, 21

Larson, R.B. 1972b, NPhS, 236, 7

Larson, R.B. 1976, MNRAS 176, 31

Lehner, N., Gry, C., Sembach, K., Hébrard, G., Chayer, P., Moos, H. W., Howk, J. C., Desert, J. M. 2002, ApJS, 140, 81

Lepine, J. R. D., \& Duvert, G. 1994, A\&A, 286, 60

López-Corredoira, M., Beckman, J. E., \& Casuso, E. 1999, A\&A, 351, 920

López-Corredoira, M., Betancort-Rijo, J., Beckman, J. E. 2002, A\&A, 386, 169

Lubowich, D. A., Pasachoff, J. M., Balonek, T. J., Millar, T. J., Tremonti, C., Roberts, H., \& Galloway, R. P. 2000, Nature, 405, 1025

Lynden-Bell, D. 1976, MNRAS, 174, 695

Madau, P., Ferguson, H. C., Dickinson, M. E., Giavalisco, M., Steidel, C. C., Fruchter, A. 1996, MNRAS, 283, 1388

Mirabel, F. 1982, ApJ, 256, 112 
Muller, C. A., Oort ,J. H., \& Raimond, E. 1963, Comptes Rendus Acad. Sci. Paris 257, 1661

Navarro, J. F., Frenk, C. S, \& White, S. 1994, MNRAS, 267, 1

Navarro, J. F., Frenk, C. S, \& White, S. 1995, MNRAS, 275, 56

Nikiforov, I. I., Petrovsky, I. V., \& Ninkova, S. 2000, in “Small Galaxy Groups” ASP conference series 209, (Eds. M. Valtonen \& C. Flynn), p. 399

Oliveira, C. M., Hébrard, G., Howk, J. C., Kruk, J. W., Chayer, P., \& Moos, H. W. 2003, ApJ, 587,235

Oort, J. H. 1970, A\&A, 7, 181

Pagel, B. E. J. 1987 in "The Galaxy” (Eds. G. Gilmore \& R. Carswell) D.Reidel, p. 341

Pagel, B. E. J., Patchett, B. E. 1975, MNRAS, 172, 13

Pérez-Ramírez, D., Knapen, J. H., Peletier, R. F. et al. 2000, MNRAS, 317, 234

Pettini, M., \& Bowen, D. V. 2001, ApJ, 560, 41

Phookun, B., Vogel, S. N., \& Mundy, L. G. 1993, ApJ, 418, 113

Power, C., Navarro, J. F., Jenkins, A., Frenk, C. S., White, S., Springel, V., Stadel, J., \& Quinn, T. 2003, MNRAS, 338, 14

Putman, M. E. et al. 2002, AJ, 123, 873

Ramaty, R., Scully, S. T., Lingenfelter, R. E., \& Kozlovsky, B., 2000, ApJ, 534, 747

Reeves H. 1974, ARA\&A, 12, 437

Regan, M., Teuben, P., Vogel, S., \& van der Hulst, T. 1996, AJ, 112, 2549

Relaño, M., Beckman, J. E., Zurita, A., \& Rozas, M. 2003, Rev. Mex.Astr Astrofis., 15, 205

Relaño, M., Beckman, J. E., Zurita, A., \& Rozas, M. 2004, A\&A, (submitted)

Reynaud, D., \& Downes D. 1997, A\&A, 319, 737

Reynaud, D., \& Downes D. 1998, A\&A, 337, 671

Reynaud, D., \& Downes D. 1999, A\&A, 347, 37

Rocha-Pinto, H. J., \&Maciel, W. J. 1996, MNRAS, 279, 447

Rocha-Pinto, H. J., Scalo, J., Maciel, W. J., \& Flynn, C. 2000, A\&A, 358, 869

Rosenberg, J. L., \& Schneider, S. E. 2002, ApJ, 567, 247

Samland, M., \& Gerhard, O.E. 2003, A\&A, 399, 961

Savage, B. D. et al. 2000, ApJS, 129, 563

Savage, B. D. et al. 2003, ApJS, 146, 165

Schmidt, M. 1963, ApJ, 137, 758

Sembach, K. R. et al. 2004, ApJS, 150, 387

Tinsley, B. 1977, ApJ, 216, 548

Tinsley, B. 1980, Fund. Cosm. Phys., 5, 287

Tomita, A., Tomita, Y., \& Saito, M. 1996, PASJ, 48, 285

Toomre, A. 1977 in "The Evolution of Galaxies and Stellar Populations" (Eds. B. Tinsley \& R. B. Larson), New Haven, Yale U. Obs., p. 401

Toomre, A., \& Toomre, J. 1972, ApJ, 178, 623

Valle, G., Ferrini, F., Galli, D., \& Shore, S. N. 2002, ApJ, 566, 252

van den Bergh, S. 1962, AJ, 67, 486

van der Hulst, T. J. M., \& Sancisi, R. 2003, in "Recycling Intergalactic and Interstellar Matter", IAU Symposium 217, p. 140

Vaughan, A., \& Preston, G. W. 1980, PASP, 92, 385

Veeraraghavan, S. \& White, S. D. M. 1985, ApJ, 296, 376

Wakker, B. P. et al. 2003, ApJS, 140, 91

Whiting, A. B. 1999, Proc. IAU Symposium 192 "The Stellar Content of the Local Group of Galaxies" (Eds. P. Whitelock \& R. Cannon), ASP, p. 420

Wielen, R. F. 1977, A\&A, 60, 263

Wielen, R. F., Fuchs, B., \& Dettbarn, C. 1996, A\&A, 314, 438

Worthey, G., Dorman, B., \& Jones, L. A. 1996, AJ, 112, 948 
Zepf, S. E., \& Koo, D. C. 1989, ApJ, 337, 34

Zurita, A., Relaño, M., Beckman, J. E., \& Knapen, J. H. 2004, A\&A, 413, 73

Zurita, A., Relaño, M., Beckman, J. E., \& Knapen, J. H. 2004a (these proceedings) 